\title{
Thermodynamics of the Rubber-Sulfur System at High Pressures ${ }^{1}$
}

\author{
Charles E. Weir
}

\begin{abstract}
From previously reported $P V T$ data on rubber-sulfur vulcanizates in the ranges $10^{\circ}$ to $80^{\circ} \mathrm{C}, 1,000$ to $10,000 \mathrm{~atm}$, and 10 to 28 percent of sulfur, calculated values are given for work, heat, internal-energy change, and difference in specific heats. These values are given at 10 -deg-C and 1,000-atm intervals for three representative rubbers containing 10, 18, and 28 percent of sulfur. Implications of the results are discussed.
\end{abstract}

\section{Introduction}

In two previous reports data have been presented on PVT measurements on rubber-sulfur vulcanizates in the ranges 10 to 28 percent of sulfur, $10^{\circ}$ to $80^{\circ} \mathrm{C}$, and 1 to $10,000 \mathrm{~atm}[1] ;^{2}$ the empirical isobaric, isothermal, and $P V T$ equations that represented the data [1]; and some interpretations of the data with regard to the isobaric glass transition temperature $\left(T_{\mathrm{g}}\right)$ and the isothermal glass transition pressure $\left(P_{\mathrm{g}}\right)$ [2]. The present report describes additional treatment of the experimental data [1] in calculation of thermodynamic quantities of interest. These calculations are patterned after those carried out by Bridgman in many studies on liquids. A résumé of data so obtained has been given by Bridgman [3]. Data are presented here for the following quantities as functions of pressure and temperature over the ranges referred to above for three rubbersulfur vulcanizates: 1 , work of compression; 2 , heat of compression; 3 , change in internal energy on compression; and 4, difference between specific heats at constant pressure and constant volume.

\section{General Considerations}

The following considerations are pertinent to the calculations carried out: All measurements carried out earlier [1] were conducted isothermally and represented measurements of relative changes of volume at various pressures. The volume was measured at one temperature $\left(21^{\circ} \mathrm{C}\right)$, initial volumes at all other temperatures being derived from the measured volume at $21^{\circ} \mathrm{C}$ and independent expansivity data. This procedure has been described in detail [1]. Theoretically, such measurements conducted isothermally at various temperatures combined with a measurement of expansitity at a single pressure are sufficient to define all states of interest here. In practice, however, the quantities required for calculation are the derivatives of the volume with respect to temperature or pressure. By virtue of the method of measurement, derivatives of volume

1 The work described in this report was sponsored by the Office of Naval Research.

${ }_{2}^{2}$ Figures in brackets indicate the literature references at the end of this paper. with respect to pressure are believed to be known to a satisfactory degree of accuracy at all temperatures. It is clear, however, that the derivatives of volume with respect to temperature are specifically known only at one pressure, atmospheric pressure. At all elevated pressures expansivities must be deduced from compression measurements and are therefore subject to all the errors included in the compression measurements. The expansivities are consequently subject to some uncertainty and any calculations dependent on the expansivities must not be considered as numerically much more significant than for order of magnitude.

It was hoped that the empirical PVT equations derived [1] would suffice for the calculations described here and thereby reduce the calculations to ordinary mathematical manipulations. This expectation was not realized and all data to be presented were obtained by graphical methods. The fact that the empirical equations may not be used in such calculations does not reflect doubt on the validity of the equations because they were derived to reproduce only the volume at various pressures and temperatures. There is no a priori reasoning that predicts that such empirical equations should also reproduce the slopes of the isobars or isotherms.

From the tabulated volume changes and volumes at $21^{\circ} \mathrm{C}$ given earlier [1] and the expansivities derived from the data of Scott [4], the specific volume was calculated at every pressure and temperature measured. From the tabular data, isobars were drawn to a large scale at every $1,000 \mathrm{~atm}$. Typical isobars of this nature have been given earlier [2]. The isobars may be considered as linear within the experimental error of the measurements and from the straight lines drawn through the experimental points, the specific volume was tabulated at 10-deg intervals.

Calculations are given here for three rubbers containing 10, 18, and 28 percent of sulfur. The sulfur contents indicated correspond to the amounts added in the milling operation [1]. Vulcanization was effected at $140^{\circ} \mathrm{C}$ for $17 \mathrm{hr}$. The first of these compounds is soft and the last one is hard at all temperatures studied; the 18-percent-sulfur vulcanizate exhibits a glass transition at approximately $48^{\circ}$ C. For the 10- and 28-percent-sulfur vulcanizates, therefore, the linear isobars are well defined 


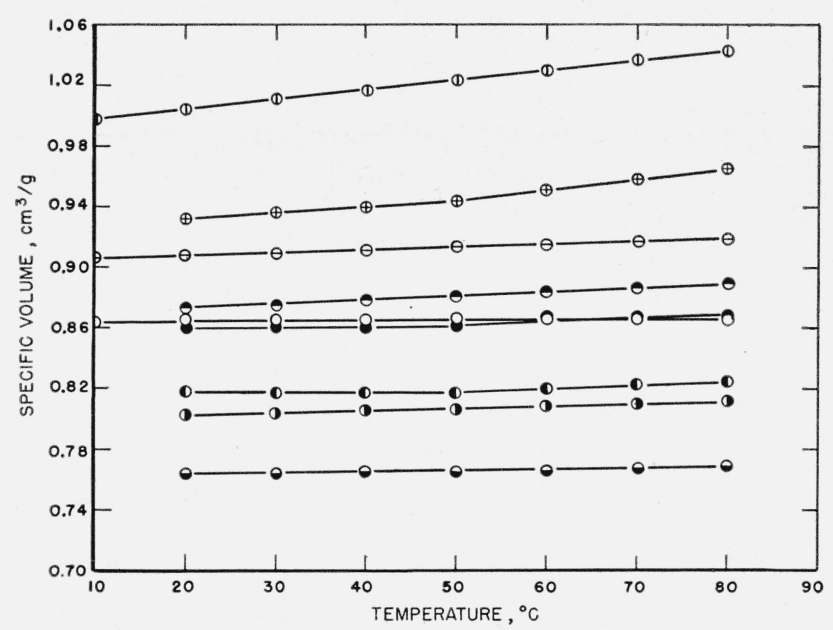

Figure 1. Typical isobars of rubber-sulfur vulcanizates.

10 percent of sulfur: $(1,1 \mathrm{~atm} ; \ominus, 5,000 \mathrm{~atm} ; \bigcirc, 10,000 \mathrm{~atm}$. 18 percent of sulfur: $\oplus, 1 \mathrm{~atm} ; \mathbf{Q}, 5,000 \mathrm{~atm} ; \mathbf{0}, 10,000 \mathrm{~atm}$. 28 percent of sulfur: $\Theta, 1 \mathrm{~atm} ; \mathbf{W}, 5,000 \mathrm{~atm} ; 10,000 \mathrm{~atm}$. because all experimental points may be considered in drawing the isobars. For the 18-percent-sulfur vulcanizate, two intersecting lines were drawn; the slopes of these lines are not as well defined because fewer experimental points determine each line. The glass transition temperature, $T_{g}$, is taken to be the temperature at which these lines intersect. The tabular specific volumes obtained in this manner are given in tables 1 to 3 , inclusive. All subsequent calculated values are derived directly from the data of these tables.

The expansivities indicated in table 3 are observed to vary with pressure in accordance with the findings of Bridgman [3] on liquids. In tables 1 and 2 , it is very probable that the pressure dependence of expansivity is somewhat too large. This conclusion results directly from the experimental data, which if taken literally indicate a negative expansivity in some instances at high pressures at the lower temperatures. This negative expansivity may be real (see Bridgman [3]); but since the experimental errors are such as to cause doubt as to its validity, the linear isobars were drawn so as to always show a positive expansivity. However, the value of expansivity thus obtained is lowered by the influence of the points contributing the negative expansivity. In this connection, it may be pointed out that the empirical equations previously derived [1] will also yield negative expansivities in certain ranges of pressure and temperature because they fit the experimental data quite well. This factor represents the principal reason why the equations were not considered suited for these calculations.

Typical specific volume-temperature and specific volume-pressure relationships are shown in figures 1 and 2.

Compressibilities to be used were also calculated directly from the data of tables 1 to 3 by means of first differences. The values so obtained appear as accurate and consistent as two graphical methods that were attempted.

All calculations were performed in experimental units of $\mathrm{cm}^{3}$-atm. The final results were then converted into joules, using the conversion relationship $1 \mathrm{j}=9.869 \mathrm{~cm}^{3}$-atm.

TABLE 1. Specific volumes of rubber-sulfur vulcanizate containing 10 percent of sulfur

\begin{tabular}{|c|c|c|c|c|c|c|c|c|}
\hline \multirow{2}{*}{ Pressure } & \multicolumn{8}{|c|}{ Specific volume at- } \\
\hline & $10^{\circ} \mathrm{C}$ & $20^{\circ} \mathrm{C}$ & $30^{\circ} \mathrm{C}$ & $40^{\circ} \mathrm{C}$ & $50^{\circ} \mathrm{C}$ & $60^{\circ} \mathrm{C}$ & $70^{\circ} \mathrm{C}$ & $80^{\circ} \mathrm{C}$ \\
\hline $\begin{array}{c}\quad a t m \\
1, \ldots 00 \\
1,000 \\
2,000 \\
3,000 \\
4,000\end{array}$ & $\begin{array}{r}c n^{3} / g \\
0.9975 \\
.9732 \\
.9485 \\
.9303 \\
.9170\end{array}$ & $\begin{array}{r}c m^{3} / g \\
1.0041 \\
.9785 \\
.9527 \\
.9338 \\
.9196\end{array}$ & $\begin{array}{c}\mathrm{cm}^{3} / \mathrm{g} \\
1.0106 \\
.9836 \\
.9570 \\
.9373 \\
.9221\end{array}$ & $\begin{array}{r}\mathrm{cm}^{3} / \mathrm{g} \\
1.0172 \\
.9890 \\
.9611 \\
.9407 \\
.9246\end{array}$ & $\begin{array}{c}c m^{3} / g \\
1.0237 \\
.9940 \\
.9652 \\
.9442 \\
.9270\end{array}$ & $\begin{array}{r}c m^{3} / g \\
1.0300 \\
.9992 \\
.9694 \\
.9477 \\
.9295\end{array}$ & $\begin{array}{c}c m^{3} / g \\
1.0365 \\
1.0043 \\
.9735 \\
.9511 \\
.9320\end{array}$ & $\begin{array}{r}\mathrm{cm}^{3} / \mathrm{g} \\
1.0430 \\
1.0094 \\
.9775 \\
.9545 \\
.9345\end{array}$ \\
\hline $\begin{array}{l}5,000 \\
6,000 \\
7,000 \\
8,000 \\
9,000\end{array}$ & $\begin{array}{l}.9060 \\
.8960 \\
.8875 \\
.8797 \\
.8725\end{array}$ & $\begin{array}{l}.9078 \\
.8975 \\
.8886 \\
.8802 \\
.8727\end{array}$ & $\begin{array}{l}.9096 \\
.8990 \\
.8896 \\
.8806 \\
.8730\end{array}$ & $\begin{array}{l}.9114 \\
.9006 \\
.8906 \\
.8810 \\
.8733\end{array}$ & $\begin{array}{l}.9132 \\
.9021 \\
.8916 \\
.8814 \\
.8736\end{array}$ & $\begin{array}{l}.9150 \\
.9035 \\
.8925 \\
.8818 \\
.8738\end{array}$ & $\begin{array}{l}.9166 \\
.9050 \\
.8934 \\
.8822 \\
.8740\end{array}$ & $\begin{array}{l}.91 \diamond 4 \\
.9065 \\
.8943 \\
.8826 \\
.8743\end{array}$ \\
\hline 10,000 & .8648 & .8649 & .8650 & .8651 & $.86 \tilde{2} 2$ & .8653 & .8654 & .8655 \\
\hline
\end{tabular}


TABLE 2. Specific volumes of rubber-sulfur vulcanizate containing 18 percent of sulfur

\begin{tabular}{|c|c|c|c|c|c|c|c|}
\hline \multirow{2}{*}{ Pressure } & \multicolumn{7}{|c|}{ Specific volume at- } \\
\hline & $20^{\circ} \mathrm{C}$ & $30^{\circ} \mathrm{C}$ & $40^{\circ} \mathrm{C}$ & $50^{\circ} \mathrm{C}$ & $60^{\circ} \mathrm{C}$ & $70^{\circ} \mathrm{C}$ & $80^{\circ} \mathrm{C}$ \\
\hline $\begin{array}{l}1, \quad \text { atm } \\
1,000 \\
2,000 \\
3,000 \\
4,000\end{array}$ & $\begin{array}{c}c m^{3} / g \\
0.9324 \\
.9163 \\
.8994 \\
.8842 \\
.8711\end{array}$ & $\begin{array}{l}c m^{3} / g \\
0.9362 \\
.9176 \\
.9000 \\
.8846 \\
.8715\end{array}$ & $\begin{array}{c}c m^{3} / g \\
0.9400 \\
.9199 \\
.9005 \\
.8850 \\
.8719\end{array}$ & $\begin{array}{r}c m^{3} / g \\
0.9444 \\
.9227 \\
.9015 \\
.8856 \\
.8724\end{array}$ & $\begin{array}{l}c m^{3} / g \\
0.9518 \\
.9288 \\
.9060 \\
.8889 \\
.8755\end{array}$ & $\begin{array}{c}c m^{3} / g \\
0.9592 \\
.9349 \\
.9106 \\
.8923 \\
.8782\end{array}$ & $\begin{array}{r}\mathrm{cm}^{3} / \mathrm{g} \\
0.9666 \\
.9410 \\
.9152 \\
.8956 \\
.8810\end{array}$ \\
\hline $\begin{array}{l}5,000 \\
6,000 \\
7,000 \\
8,000 \\
9,000\end{array}$ & $\begin{array}{l}.8599 \\
.8490 \\
.8402 \\
.8320 \\
.8249\end{array}$ & $\begin{array}{l}.8602 \\
.8494 \\
.8405 \\
.8321 \\
.8252\end{array}$ & $\begin{array}{l}.8604 \\
.8498 \\
.8408 \\
.8321 \\
.8253\end{array}$ & $\begin{array}{l}.8606 \\
.8505 \\
.8413 \\
.8322 \\
.8255\end{array}$ & $\begin{array}{l}.8634 \\
.8534 \\
.8438 \\
.8345 \\
.8274\end{array}$ & $\begin{array}{l}.8661 \\
.8563 \\
.8465 \\
.8369 \\
.8297\end{array}$ & $\begin{array}{l}.8689 \\
.8592 \\
.8490 \\
.8393 \\
.8320\end{array}$ \\
\hline 10,000 . & .8170 & .8171 & .8172 & .8176 & .8200 & .8224 & .8246 \\
\hline
\end{tabular}

TABLE 3. Specific volumes of rubber-sulfur vulcanizate containing 28 percent of sulfur

\begin{tabular}{|c|c|c|c|c|c|c|c|}
\hline \multirow{2}{*}{ Pressure } & \multicolumn{7}{|c|}{ Specific volume at- } \\
\hline & $20^{\circ} \mathrm{C}$ & $30^{\circ} \mathrm{C}$ & $40^{\circ} \mathrm{C}$ & $50^{\circ} \mathrm{C}$ & $60^{\circ} \mathrm{C}$ & $70^{\circ} \mathrm{C}$ & $80^{\circ} \mathrm{C}$ \\
\hline $\begin{array}{l}\quad \quad \text { atm } \\
1, \ldots 00 \\
2,000 \\
3,000 \\
4,000\end{array}$ & $\begin{array}{l}c m^{3} / g \\
0.8730 \\
.8563 \\
.8400 \\
.8260 \\
.8130\end{array}$ & $\begin{array}{l}c m^{3} / g \\
0.8758 \\
.8588 \\
.8422 \\
.8277 \\
.8149\end{array}$ & $\begin{array}{l}c m^{3} / g \\
0.8786 \\
.8613 \\
.8444 \\
.8295 \\
.8168\end{array}$ & $\begin{array}{l}\mathrm{cm}^{3} / \mathrm{g} \\
0.8814 \\
.8637 \\
.8468 \\
.810 \\
.8187\end{array}$ & $\begin{array}{l}c m^{3} / g \\
0.8840 \\
.8660 \\
.8485 \\
.8326 \\
.8205\end{array}$ & $\begin{array}{l}c m^{3} / g \\
0.8867 \\
.8683 \\
.8505 \\
.8343 \\
.8223\end{array}$ & $\begin{array}{c}c m^{3} / g \\
0.8895 \\
.8706 \\
.8525 \\
.8359 \\
.8240\end{array}$ \\
\hline $\begin{array}{l}5,000 \\
6,000 \\
7,000 \\
8,000 \\
9,000\end{array}$ & $\begin{array}{l}.8029 \\
.7931 \\
.7842 \\
.7761 \\
.7697\end{array}$ & $\begin{array}{l}.8045 \\
.7948 \\
.7860 \\
.7774 \\
.7710\end{array}$ & $\begin{array}{r}.8060 \\
.7964 \\
.7873 \\
.7786 \\
.7722\end{array}$ & $\begin{array}{l}.8075 \\
.7980 \\
.7886 \\
.7798 \\
.7733\end{array}$ & $\begin{array}{l}.8090 \\
.7996 \\
.7900 \\
.7810 \\
.7745\end{array}$ & $\begin{array}{l}.8105 \\
.8012 \\
.7913 \\
.7822 \\
.7757\end{array}$ & $\begin{array}{l}.8120 \\
.8027 \\
.7925 \\
.7834 \\
.7768\end{array}$ \\
\hline 10,000 & .7628 & .7639 & .7650 & .7660 & .7671 & .7683 & .7694 \\
\hline
\end{tabular}

\section{Work of Compression}

The work of compression defined as

$$
W=\int_{P_{1}}^{P_{2}} P\left(\frac{\partial V}{\partial P}\right)_{T} d P
$$

was calculated by graphical integration of the pressure-volume data of tables 1 to 3 by mechanical methods. The values obtained are given in tables 4 to 6 . These data represent work done on the specimen by the external compressive forces. The work is observed to be only slightly affected by temperature, but strongly pressure dependent. Graphs of work as a function of pressure show the approximate linearity at high pressures noted by Bridgman [3]. It would appear from these data, however, that too much curvature still exists to permit successful use of a linear relationship for extrapolation [3]. The work of compression decreases with increasing sulfur content as expected. Such decrease is due directly to the lower compressibility of the higher sulfur content rubbers. It is to be noted that mathematical integrations of the appropriate partial derivatives of the empirical equations [1] yield results in good agreement with the data of tables 4 to 6 that were obtained graphically. Thus the empirical equations may be considered to represent both the compres-
TABLE 4. Work of compression of rubber-sulfur vulcanizate containing 10 percent of sulfur

\begin{tabular}{|c|c|c|c|c|c|c|c|c|}
\hline \multirow{2}{*}{$\underset{P}{\text { Pressure, }}$} & \multicolumn{8}{|c|}{ Work of compression between $1 \mathrm{~atm}$ and $P$ at- } \\
\hline & $10^{\circ} \mathrm{C}$ & $20^{\circ} \mathrm{C}$ & $30^{\circ} \mathrm{C}$ & $40^{\circ} \mathrm{C}$ & $50^{\circ} \mathrm{C}$ & $60^{\circ} \mathrm{C}$ & $70^{\circ} \mathrm{C}$ & $80^{\circ} \mathrm{C}$ \\
\hline $\begin{array}{c}\text { atm } \\
1,000 \ldots \\
2,000 \ldots \\
3,000 \\
4,000 \ldots \\
5,000 \ldots\end{array}$ & $\begin{array}{r}j / g \\
1.4 \\
4.8 \\
9.5 \\
14.1 \\
19.1\end{array}$ & $\begin{array}{r}j / g \\
1.4 \\
5.0 \\
9.7 \\
14.7 \\
19.9\end{array}$ & $\begin{array}{r}J / g \\
1.4 \\
5.3 \\
10.6 \\
15.9 \\
21.4\end{array}$ & $\begin{array}{r}j / g \\
1.4 \\
5.4 \\
11.0 \\
16.6 \\
22.6\end{array}$ & $\begin{array}{r}j / g \\
1.5 \\
5.6 \\
11.4 \\
17.8 \\
23.8\end{array}$ & \begin{tabular}{r}
\multicolumn{1}{c}{$j / g$} \\
1.5 \\
5.7 \\
11.8 \\
18.2 \\
24.5
\end{tabular} & \begin{tabular}{r}
\multicolumn{1}{c}{$j / g$} \\
1.6 \\
5.8 \\
12.2 \\
18.8 \\
25.7
\end{tabular} & $\begin{array}{r}j / 0 \\
1.6 \\
6.2 \\
12.7 \\
19.7 \\
26.9\end{array}$ \\
\hline $\begin{array}{l}6,000 \ldots \\
7,000 \\
8,000 \\
9,000 \\
10,000\end{array}$ & $\begin{array}{l}24.7 \\
30.3 \\
36.4 \\
42.8 \\
50.0\end{array}$ & $\begin{array}{l}25.6 \\
31.6 \\
37.8 \\
44.7 \\
52.0\end{array}$ & $\begin{array}{l}27.5 \\
33.8 \\
40.1 \\
47.5 \\
55.2\end{array}$ & $\begin{array}{l}28.6 \\
35.2 \\
41.6 \\
48.9 \\
56.6\end{array}$ & $\begin{array}{l}30.2 \\
36.9 \\
43.9 \\
51.7 \\
59.7\end{array}$ & $\begin{array}{l}31.3 \\
38.0 \\
44.8 \\
52.9 \\
61.0\end{array}$ & $\begin{array}{l}32.5 \\
39.6 \\
47.0 \\
55.2 \\
63.8\end{array}$ & $\begin{array}{l}33.9 \\
41.2 \\
49.0 \\
57.7 \\
65.5\end{array}$ \\
\hline
\end{tabular}

TABLE 5. Work of compression of rubber-sulfur vulcanizate containing 18 percent of sulfur

\begin{tabular}{|c|c|c|c|c|c|c|c|}
\hline \multirow{2}{*}{ Pressure, $P$} & \multicolumn{7}{|c|}{ Work of compression between $1 \mathrm{~atm}$ and $P$ at- } \\
\hline & $20^{\circ} \mathrm{C}$ & $30^{\circ} \mathrm{C}$ & $40^{\circ} \mathrm{C}$ & $50^{\circ} \mathrm{C}$ & $60^{\circ} \mathrm{C}$ & $70^{\circ} \mathrm{C}$ & $80^{\circ} \mathrm{C}$ \\
\hline $\begin{array}{c}\quad \text { atm } \\
1,000 \ldots \\
2,000 \\
3,000 \\
4,000 \\
5,000\end{array}$ & $\begin{array}{r}j / g \\
1.0 \\
3.4 \\
7.2 \\
11.8 \\
17.0\end{array}$ & $\begin{array}{r}j / g \\
1.0 \\
3.5 \\
7.3 \\
11.9 \\
17.4\end{array}$ & \begin{aligned} \multicolumn{1}{c}{$j / g$} \\
1.1 \\
3.6 \\
7.8 \\
12.1 \\
17.5\end{aligned} & $\begin{array}{r}j / g \\
1.3 \\
4.1 \\
8.0 \\
12.8 \\
18.2\end{array}$ & $\begin{array}{r}j / g \\
1.4 \\
4.1 \\
7.9 \\
12.6 \\
17.9\end{array}$ & $\begin{array}{r}j / g \\
1.4 \\
4.2 \\
8.0 \\
12.8 \\
18.1\end{array}$ & $\begin{array}{r}j / g \\
1.4 \\
5.8 \\
10.6 \\
16.5 \\
22.6\end{array}$ \\
\hline $\begin{array}{l}6,000 \\
7,000 \\
8,000 \\
9,000 \\
10,000\end{array}$ & $\begin{array}{l}22.9 \\
28.7 \\
35.1 \\
41.5 \\
48.2\end{array}$ & $\begin{array}{l}23.2 \\
29.1 \\
35.3 \\
42.1 \\
49.2\end{array}$ & $\begin{array}{l}23.3 \\
29.3 \\
35.5 \\
42.2 \\
49.4\end{array}$ & $\begin{array}{l}23.9 \\
30.0 \\
36.2 \\
43.0 \\
50.1\end{array}$ & $\begin{array}{l}23.9 \\
30.3 \\
36.9 \\
44.0 \\
51.6\end{array}$ & $\begin{array}{l}23.9 \\
29.9 \\
36.8 \\
43.8 \\
51.5\end{array}$ & $\begin{array}{l}28.6 \\
35.0 \\
42.2 \\
49.3 \\
56.7\end{array}$ \\
\hline
\end{tabular}

TABLE 6. Work of compression of rubber-sulfur vulcanizate containing 28 percent of sulfur

\begin{tabular}{c|r|r|r|r|r|r|r}
\hline \hline \multirow{2}{*}{ Pressure, $P$} & \multicolumn{6}{|c}{ Work of compression between 1 atm and $P$ at- } \\
\cline { 2 - 8 } & $20^{\circ} \mathrm{C}$ & $30^{\circ} \mathrm{C}$ & $40^{\circ} \mathrm{C}$ & $50^{\circ} \mathrm{C}$ & $60^{\circ} \mathrm{C}$ & $70^{\circ} \mathrm{C}$ & $80^{\circ} \mathrm{C}$ \\
\hline & $j / g$ & $j / g$ & $j / g$ & $j / g$ & $j / g$ & $j / g$ & $j / g$ \\
1,000 & 0.9 & 0.9 & 1.0 & 0.9 & 1.0 & 1.0 & 1.0 \\
2,000 & 3.3 & 3.6 & 3.6 & 3.6 & 3.7 & 3.7 & 3.8 \\
3,000 & 7.0 & 7.3 & 7.4 & 7.5 & 7.6 & 7.8 & 7.9 \\
4,000 & 11.5 & 11.8 & 11.8 & 12.2 & 12.3 & 12.4 & 12.3 \\
5,000 & 16.5 & 16.8 & 16.9 & 17.3 & 17.4 & 17.4 & 17.8 \\
6,000 & 21.9 & 22.4 & 22.6 & 22.9 & 23.3 & 23.4 & 23.5 \\
7,000 & 27.8 & 28.2 & 28.5 & 28.9 & 29.4 & 29.4 & 29.5 \\
8,000 & 33.7 & 34.2 & 34.5 & 35.1 & 35.6 & 35.6 & 35.9 \\
9,000 & 49.9 & 41.6 & 42.4 & 42.4 & 42.9 \\
10,000 & 46.9 & 47.4 & 48.0 & 48.7 & 49.7 & 49.8 & 50.3 \\
\hline
\end{tabular}

sibility and the temperature dependence of the compressibility fairly closly. It will also be noted in table 5 that no discontinuity in the work of compression occurs at $T_{\mathrm{g}}$. This result follows directly from the previous report [2] in which no discontinuity in $(\partial V / \partial P)_{T}$ was observed at $T_{\mathrm{g}}$.

\section{Heat of Compression}

The heat of compression defined as

$$
Q=-T \int_{P_{1}}^{P_{2}}\left(\frac{\partial V}{\partial T}\right)_{P} d P
$$

was calculated by graphical integration of a graph of $T(\partial V / \partial T)_{P}$ versus $P$ by mechanical methods. 
The values so obtained are given in tables 7 to 9 . These data, which are all intrinsically negative, represent the heat given off by the specimens when compressed. As noted previously, the expansivities are not known to a high accuracy and the data of tables 7 to 9 must not be interpreted too literally. In particular, the apparent excessive decrease of expansivity with increasing pressure is responsible for the low values of heat shown in table 8 at low pressures. The marked increase of heat also ob-

TABLE 7. Heat of compression of rubber-sulfur vulcanizate containing 10 percent of sulfur

\begin{tabular}{|c|c|c|c|c|c|c|c|c|}
\hline \multirow{2}{*}{$\underset{P}{\text { Pressure, }}$} & \multicolumn{8}{|c|}{ Heat of compression between $1 \mathrm{~atm}$ and $P$ at- } \\
\hline & $10^{\circ} \mathrm{C}$ & $20^{\circ} \mathrm{C}$ & $30^{\circ} \mathrm{C}$ & $40^{\circ} \mathrm{C}$ & $50^{\circ} \mathrm{C}$ & $60^{\circ} \mathrm{C}$ & $70^{\circ} \mathrm{C}$ & $80^{\circ} \mathrm{C}$ \\
\hline $\begin{array}{r}\text { atm } \\
1,000 \\
2,000 \\
3,000 \\
4,000 \\
5,000 \\
\end{array}$ & $\begin{array}{l}j / g \\
17.1 \\
31.1 \\
42.0 \\
50.2 \\
56.3\end{array}$ & $\begin{array}{l}j / g \\
18.0 \\
32.4 \\
43.8 \\
52.3 \\
58.5\end{array}$ & $\begin{array}{l}j / g \\
18.7 \\
33.7 \\
45.6 \\
54.5 \\
61.1\end{array}$ & $\begin{array}{l}j / g \\
19.4 \\
34.8 \\
46.9 \\
56.4 \\
63.4\end{array}$ & $\begin{array}{l}j / g \\
20.0 \\
36.0 \\
48.5 \\
58.2 \\
65.4\end{array}$ & $\begin{array}{l}j / g \\
20.6 \\
37.3 \\
50.2 \\
60.2 \\
67.6\end{array}$ & $\begin{array}{l}j / g \\
21.3 \\
38.5 \\
51.7 \\
62.0 \\
69.7\end{array}$ & $\begin{array}{l}j / g \\
22.0 \\
39.7 \\
53.3 \\
63.9 \\
71.9\end{array}$ \\
\hline $\begin{array}{l}6,000 \\
7,000 \\
8,000 \\
9,000 \\
10,000\end{array}$ & $\begin{array}{l}60.8 \\
63.9 \\
66.0 \\
67.3 \\
67.9\end{array}$ & $\begin{array}{l}63.3 \\
66.6 \\
68.9 \\
70.3 \\
70.9\end{array}$ & $\begin{array}{l}65.9 \\
69.4 \\
71.9 \\
73.5 \\
74.1\end{array}$ & $\begin{array}{l}68.5 \\
72.2 \\
74.8 \\
76.4 \\
77.1\end{array}$ & $\begin{array}{l}70.7 \\
74.6 \\
77.4 \\
79.3 \\
80.0\end{array}$ & $\begin{array}{l}73.0 \\
77.0 \\
81.5 \\
83.4 \\
84.2\end{array}$ & $\begin{array}{l}75.4 \\
79.6 \\
84.2 \\
86.4 \\
87.1\end{array}$ & $\begin{array}{l}77.8 \\
82.1 \\
87.0 \\
89.3 \\
90.1\end{array}$ \\
\hline
\end{tabular}

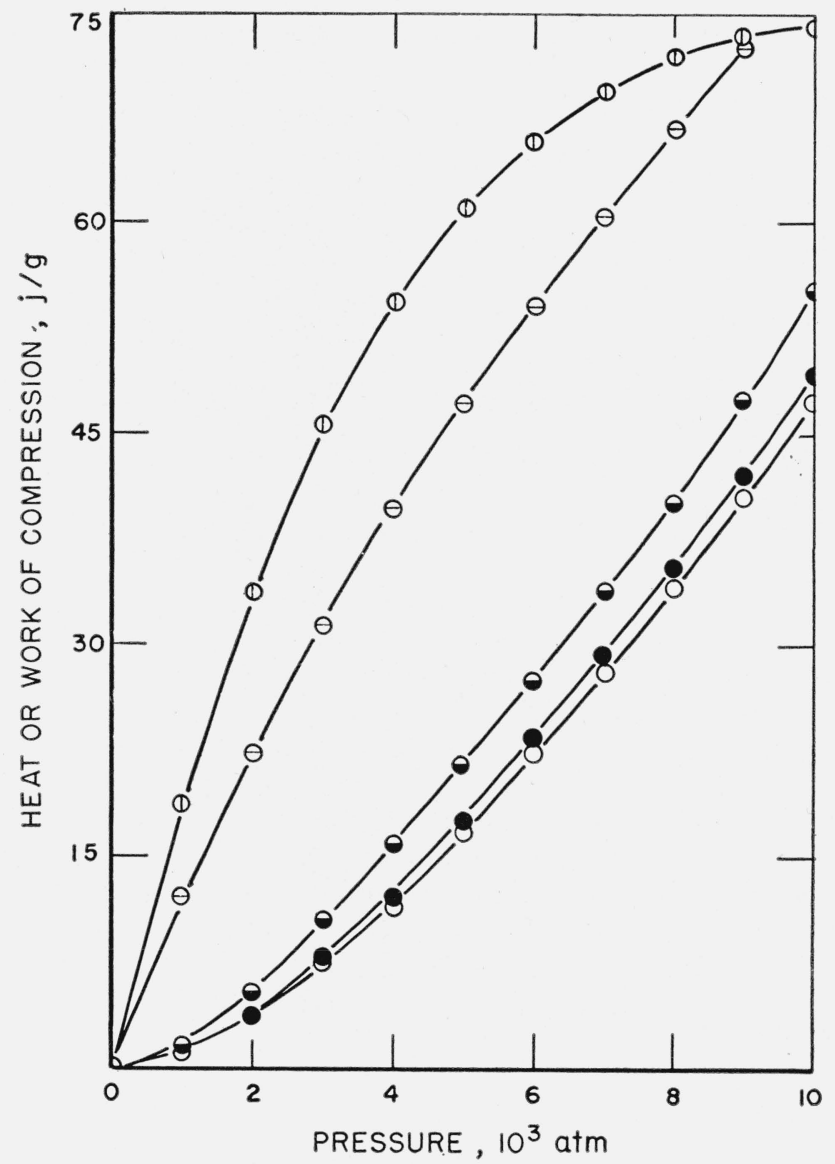

FIGURE 3. Work and heat of compression of rubber-sulfur vulcanizates at $30^{\circ} \mathrm{C}$.

10 percent of sulfur: $\Phi$, heat of compression; $\ominus$, work of compression.

18 percent of sulfur: $\mathbf{0}$, work of compression

28 percent of sulfur: $\Theta$, heat of compression; $\bigcirc$, work of compression.
TABLE 8. Heat of compression of rubber-sulfur vulcanisate containing 18 percent of sulfur

\begin{tabular}{|c|c|c|c|c|c|c|c|}
\hline \multirow{2}{*}{ Pressure, $P$} & \multicolumn{7}{|c|}{ Heat of compression between $1 \mathrm{~atm}$ and $P$ at- } \\
\hline & $20^{\circ} \mathrm{C}$ & $39^{\circ} \mathrm{C}$ & $40^{\circ} \mathrm{C}$ & $50^{\circ} \mathrm{C}$ & $60^{\circ} \mathrm{C}$ & $70^{\circ} \mathrm{C}$ & $80^{\circ} \mathrm{C}$ \\
\hline atm & $j / g$ & $j / g$ & $j / g$ & $j / g$ & $j / g$ & $j / g$ & $j / g$ \\
\hline 2,000 & & 10.1 & $\begin{array}{r}10.6 \\
\end{array}$ & 36.6 & $\begin{array}{l}1.0 \\
38.0\end{array}$ & 39.2 & 40.2 \\
\hline $3,000_{-}$ & 11. 4 & 11.8 & 12. 3 & 49. 2 & 51.4 & 53. 2 & 54.7 \\
\hline 4,000 & 12. 5 & 13. 1 & 13. 6 & 59.6 & 62.4 & 64.8 & 66. 9 \\
\hline 5,000 & 13.1 & 13.8 & 14. 3 & 69.0 & 72.4 & 75.3 & 78.0 \\
\hline 6,000 & 13.6 & 14. 6 & 15. 0 & 77. 5 & 81.4 & 85.0 & 88. 2 \\
\hline & 14. 1 & 15. 1 & 15. 6 & 85.8 & 90.4 & 94.4 & 97.9 \\
\hline 8,00 & 14. 6 & 15. 7 & 16. 2 & 93.9 & 99.0 & 103.5 & 107.3 \\
\hline 0 & 15.0 & 16. 2 & 16. 6 & 101. 4 & 107. 4 & 112.3 & 116.1 \\
\hline 10,0 & 15.4 & 16. 6 & 17.0 & 109.4 & 115.8 & 121. 2 & 124.9 \\
\hline
\end{tabular}

TABLE 9. Heat of compression of rubber-sulfur vulcanizate containing 28 percent of sulfur

\begin{tabular}{|c|c|c|c|c|c|c|c|}
\hline \multirow{2}{*}{ Pressure, $P$} & \multicolumn{7}{|c|}{ Heat of compression between $1 \mathrm{~atm}$ and $P$ at - } \\
\hline & $20^{\circ} \mathrm{C}$ & $30^{\circ} \mathrm{C}$ & $40^{\circ} \mathrm{C}$ & $50^{\circ} \mathrm{C}$ & $60^{\circ} \mathrm{C}$ & $70^{\circ} \mathrm{C}$ & $80^{\circ} \mathrm{C}$ \\
\hline $\begin{array}{l}\quad \text { atm } \\
1,000 \\
2,000 \\
3,000 \\
4,000 \\
5,000\end{array}$ & $\begin{array}{l}j / g \\
11.6 \\
21.5 \\
30.2 \\
38.0 \\
45.3\end{array}$ & $\begin{array}{l}j / g \\
12.0 \\
22.3 \\
31.4 \\
39.6 \\
47.1\end{array}$ & $\begin{array}{l}j / g \\
12.5 \\
23.2 \\
32.5 \\
41.0 \\
48.9\end{array}$ & $\begin{array}{l}j / g \\
13.0 \\
24.0 \\
33.7 \\
42.5 \\
50.6\end{array}$ & $\begin{array}{l}j / g \\
13.4 \\
24.7 \\
34.7 \\
43.8 \\
52.1\end{array}$ & $\begin{array}{l}j / g \\
13.6 \\
25.3 \\
35.6 \\
44.9 \\
53.6\end{array}$ & $\begin{array}{l}j / g \\
\quad 14.0 \\
26.0 \\
36.6 \\
46.2 \\
55.1\end{array}$ \\
\hline $\begin{array}{l}6,000 \\
7,000 \\
8,000 \\
9,000 \\
10,000\end{array}$ & $\begin{array}{l}52.1 \\
58.5 \\
64.5 \\
70.2 \\
75.5\end{array}$ & $\begin{array}{l}54.2 \\
60.7 \\
66.9 \\
72.6 \\
78.1\end{array}$ & $\begin{array}{l}56.2 \\
62.9 \\
69.3 \\
75.2 \\
80.6\end{array}$ & $\begin{array}{l}58.1 \\
65.1 \\
71.6 \\
77.7 \\
83.3\end{array}$ & $\begin{array}{l}59.9 \\
67.1 \\
73.9 \\
80.2 \\
86.0\end{array}$ & $\begin{array}{l}61.6 \\
69.0 \\
76.0 \\
82.6 \\
88.7\end{array}$ & $\begin{array}{l}63.4 \\
71.1 \\
78.3 \\
85.1 \\
91.5\end{array}$ \\
\hline
\end{tabular}

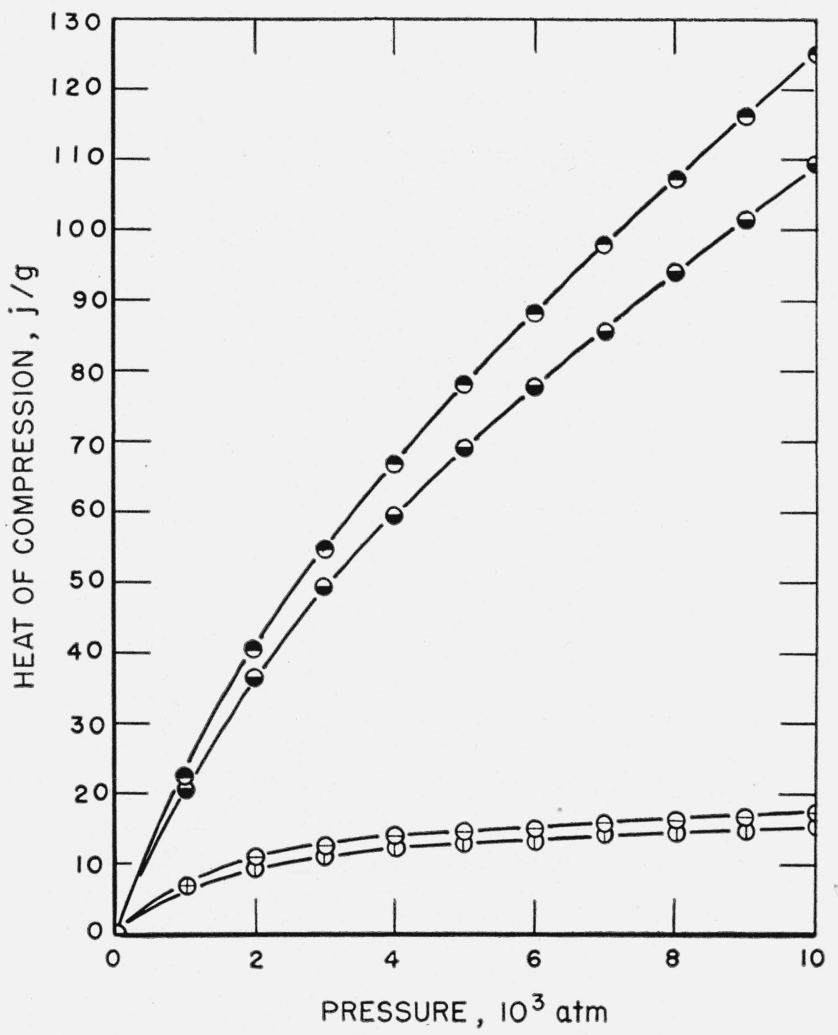

FigURE 4. Heat of compression of rubber-sulfur vuicanizate containing 18 percent of sulfur.

$\boldsymbol{\ominus}, 80^{\circ} \mathrm{C} ; \boldsymbol{\ominus}, 50^{\circ} \mathrm{C} ; \ominus, 40^{\circ} \mathrm{C} ; \oplus, 20^{\circ} \mathrm{C}$. 
served in table 8 at temperatures above $T_{\mathbf{g}}$ is real and follows necessarily from the increased expansivity above $T_{\mathrm{g}}$.

Typical data for work and heat of compression are shown in figures 3 and 4.

\section{Internal Energy Change on Compression}

The internal energy change on compression is obtained by subtracting corresponding values of heat of compression from work of compression, considering both values as intrinsically positive. The results obtained are shown in tables 10 to 12 . Any discrepancies noted in results obtained from subtracting data given here in tables 4 to 6 and 7 to 9 with the data of tables 10 to 12 arises from the fact that all calculations were performed initially in experimental units of $\mathrm{cm}^{3}-\mathrm{atm} / \mathrm{g}$ before converting to the units reported here. Considering first the results of tables 10 and 12 , which may be considered to be essentially numerically correct for reasons discussed earlier, it is noted that on isothermal compression the internal energy initially decreases and at an elevated pressure starts to increase with increasing pressure. This behavior has been noted by Bridgman [3], who has offered the explanation that under ordinary conditions the molecular forces are predominately attractive so that on compression the heat liberated initially overcompensates the work

TABLE 10. Change in internal energy on compression of rubbersulfur vulcanizate containing 10 percent of sulfur

\begin{tabular}{|c|c|c|c|c|c|c|c|c|}
\hline \multirow{2}{*}{$\begin{array}{c}\text { Pressure, } \\
P\end{array}$} & \multicolumn{8}{|c|}{ Change in internal energy between $1 \mathrm{~atm}$ and $P$ at- } \\
\hline & $10^{\circ} \mathrm{C}$ & $20^{\circ} \mathrm{C}$ & $30^{\circ} \mathrm{C}$ & $40^{\circ} \mathrm{C}$ & $50^{\circ} \mathrm{C}$ & $60^{\circ} \mathrm{C}$ & $70^{\circ} \mathrm{C}$ & $80^{\circ} \mathrm{C}$ \\
\hline $\begin{array}{r}\text { atm } \\
1,000 \\
2,000\end{array}$ & $\begin{array}{c}j / g \\
-15.8 \\
-26.2 \\
-32.5 \\
-36.2 \\
-37.3\end{array}$ & $\begin{array}{c}j / g \\
-16.6 \\
-27.4 \\
-34.0 \\
-37.6 \\
-38.7\end{array}$ & $\begin{array}{c}j / g \\
-17.2 \\
-28.4 \\
-34.8 \\
-38.6 \\
-39.7\end{array}$ & $\begin{array}{c}j / g \\
-17.9 \\
-29.3 \\
-36.0 \\
-39.8 \\
-40.8\end{array}$ & $\begin{array}{c}j / g \\
-18.4 \\
-30.4 \\
-37.1 \\
-40.4 \\
-41.7\end{array}$ & $\begin{array}{c}j / g \\
-20.2 \\
-31.6 \\
-38.4 \\
-42.0 \\
-43.1\end{array}$ & $\begin{array}{c}j / g \\
-19.8 \\
-32.6 \\
-39.5 \\
-43.3 \\
-44.0\end{array}$ & $\begin{array}{l}j / g \\
-20.4 \\
-33.4 \\
-40.6 \\
-44.2 \\
-45.0\end{array}$ \\
\hline $\begin{array}{l}6,000 \\
7,000 \\
8,000 \\
9,000 \\
10,000\end{array}$ & $\begin{array}{l}-36.2 \\
-33.6 \\
-29.6 \\
-24.4 \\
-17.8\end{array}$ & $\begin{array}{l}-37.7 \\
-35.2 \\
-31.1 \\
-25.6 \\
-18.9\end{array}$ & $\begin{array}{l}-38.4 \\
-35.6 \\
-31.8 \\
-26.1 \\
-19.0\end{array}$ & $\begin{array}{l}-39.8 \\
-37.0 \\
-33.1 \\
-27.6 \\
-20.5\end{array}$ & $\begin{array}{l}-40.5 \\
-37.7 \\
-33.4 \\
-27.6 \\
-20.3\end{array}$ & $\begin{array}{l}-41.6 \\
-38.9 \\
-36.7 \\
-30.6 \\
-23.2\end{array}$ & $\begin{array}{l}-42.9 \\
-40.0 \\
-37.2 \\
-31.2 \\
-23.3\end{array}$ & $\begin{array}{l}-43.9 \\
-40.9 \\
-37.9 \\
-31.5 \\
-23.6\end{array}$ \\
\hline
\end{tabular}

TABLE 11. Change in internal energy on compression of rubbersulfur vulcanizate containing 18 percent of sulfur

\begin{tabular}{|c|c|c|c|c|c|c|c|}
\hline \multirow{2}{*}{ Pressure, $P$} & \multicolumn{7}{|c|}{ Change in internal energy between $1 \mathrm{~atm}$ and $P$ at- } \\
\hline & $20^{\circ} \mathrm{C}$ & $30^{\circ} \mathrm{C}$ & $40^{\circ} \mathrm{C}$ & $50^{\circ} \mathrm{C}$ & $60^{\circ} \mathrm{C}$ & $70^{\circ} \mathrm{C}$ & $80^{\circ} \mathrm{C}$ \\
\hline $\begin{array}{c}\quad \text { atm } \\
1,000 \\
2,000 \\
3,000 \\
4,000 \\
5,000\end{array}$ & $\begin{array}{l}j / g \\
-6.0 \\
-6.7 \\
-4.2 \\
-0.7 \\
+3.9\end{array}$ & $\begin{array}{l}j / g \\
-6.1 \\
-6.6 \\
-4.5 \\
-1.2 \\
+3.5\end{array}$ & $\begin{array}{l}j / g \\
-6.3 \\
-6.9 \\
-4.4 \\
-1.5 \\
+3.2\end{array}$ & $\begin{aligned} & j / g \\
&-19.5 \\
&-32.5 \\
&-41.2 \\
&-46.8 \\
&-50.8\end{aligned}$ & $\begin{array}{l}j / g \\
-20.3 \\
-33.9 \\
-43.4 \\
-49.8 \\
-54.4\end{array}$ & $\begin{aligned} & j / g \\
&-20.8 \\
&-34.9 \\
&-45.1 \\
&-52.0 \\
&-57.2\end{aligned}$ & $\begin{array}{l}j / g \\
-21.4 \\
-34.4 \\
-44.1 \\
-50.5 \\
-55.4\end{array}$ \\
\hline $\begin{array}{l}6,000 \\
7,000 \\
8,000 \\
9,000 \\
10,000\end{array}$ & $\begin{array}{r}+9.2 \\
+14.6 \\
+20.5 \\
+26.5 \\
+32.9\end{array}$ & $\begin{array}{r}+8.6 \\
+14.0 \\
+19.6 \\
+25.9 \\
+31.6\end{array}$ & $\begin{array}{r}+8.3 \\
+13.8 \\
+19.3 \\
+25.6 \\
+32.3\end{array}$ & $\begin{array}{l}-53.6 \\
-55.8 \\
-57.6 \\
-58.4 \\
-59.3\end{array}$ & $\begin{array}{l}-57.6 \\
-60.1 \\
-62.2 \\
-63.4 \\
-64.3\end{array}$ & $\begin{array}{l}-61.2 \\
-64.6 \\
-66.7 \\
-68.5 \\
-70.7\end{array}$ & $\begin{array}{l}-59.6 \\
-62.9 \\
-65.1 \\
-66.8 \\
-68.2\end{array}$ \\
\hline
\end{tabular}

TABLE 12. Change in internal energy on compression of rubbersulfur vulcanizate containing 28 percent of sulfur

\begin{tabular}{|c|c|c|c|c|c|c|c|}
\hline \multirow{2}{*}{ Pressure, $P$} & \multicolumn{7}{|c|}{ Change in internal energy between $1 \mathrm{~atm}$ and $P$ at- } \\
\hline & $20^{\circ} \mathrm{C}$ & $30^{\circ} \mathrm{C}$ & $40^{\circ} \mathrm{C}$ & $50^{\circ} \mathrm{C}$ & $60^{\circ} \mathrm{C}$ & $70^{\circ} \mathrm{C}$ & $80^{\circ} \mathrm{C}$ \\
\hline $\begin{array}{l}\text { atm } \\
1,000 \\
2,000 \\
3,000 \\
4,000 \\
5,000\end{array}$ & $\begin{array}{c}j / g \\
-10.6 \\
-18.2 \\
-23.1 \\
-26.4 \\
-28.9\end{array}$ & $\begin{array}{c}j / g \\
-11.1 \\
-18.7 \\
-24.1 \\
-27.7 \\
-30.3\end{array}$ & $\begin{array}{c}j / g \\
-11.6 \\
-19.8 \\
-25.1 \\
-29.2 \\
-31.9\end{array}$ & $\begin{array}{c}j / g \\
-12.0 \\
-20.5 \\
-26.2 \\
-30.4 \\
-33.2\end{array}$ & $\begin{array}{c}j / g \\
-12.4 \\
-21.1 \\
-27.0 \\
-31.4 \\
-34.7\end{array}$ & $\begin{array}{c}j / g \\
-12.6 \\
-21.5 \\
-27.8 \\
-32.5 \\
-36.2\end{array}$ & $\begin{array}{l}j / g \\
-13.0 \\
-22.2 \\
-28.7 \\
-33.9 \\
-37.3\end{array}$ \\
\hline $\begin{array}{l}6,000 \\
7,000 \\
8,000 \\
9,000 \\
10,000\end{array}$ & $\begin{array}{l}-30.7 \\
-30.7 \\
-30.8 \\
-30.3 \\
-29.1\end{array}$ & $\begin{array}{l}-31.6 \\
-32.5 \\
-32.6 \\
-32.1 \\
-30.6\end{array}$ & $\begin{array}{l}-33.5 \\
-34.4 \\
-34.8 \\
-34.2 \\
-32.6\end{array}$ & $\begin{array}{l}-35.2 \\
-36.2 \\
-36.6 \\
-36.1 \\
-34.6\end{array}$ & $\begin{array}{l}-36.6 \\
-37.7 \\
-38.3 \\
-37.8 \\
-36.4\end{array}$ & $\begin{array}{l}-38.2 \\
-39.7 \\
-40.5 \\
-40.1 \\
-36.8\end{array}$ & $\begin{array}{l}-39.8 \\
-41.5 \\
-42.4 \\
-42.2 \\
-41.2\end{array}$ \\
\hline
\end{tabular}

performed on compression. At some elevated pressure, however, this process must reverse because the forces must ultimately become repulsive. Therefore, it is to be expected that at some pressure above the experimental range, the internal energy change will reverse sign and become positive. This pressure of reversal cannot be reliably estimated on the basis of these data. It is to be noted, however, that the pressure of reversal on the basis of these data increases with increasing temperature as required by Bridgman's [3] explanation and also increases with increasing hardness of rubber. The data of table 11 are more interesting. The marked change in internal energy change on passing through the thermal transition is to be noted. This change, while numerically uncertain, is of unquestionable validity since $(\partial V / \partial T)_{P}$ changes markedly at $T_{\mathrm{g}}$, whereas $(\partial V / \partial P)_{T}$ does not. The low pressure at which the internal energy change reverses sign at

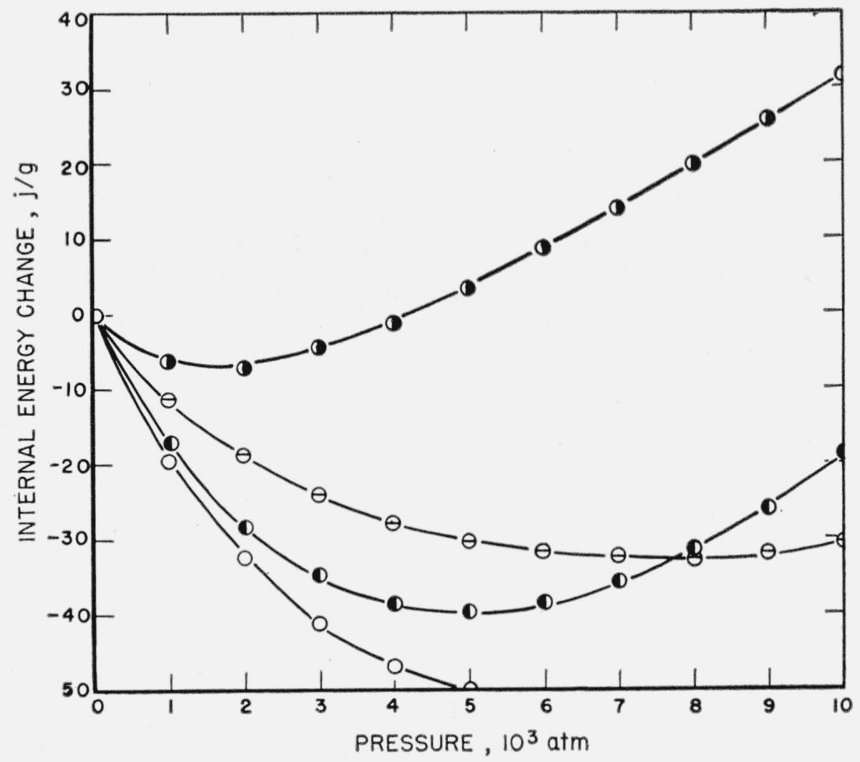

FIGURE 5. Typical isothermal internal-energy-change behavior.

10 percent of sulfur at $30^{\circ} \mathrm{C}, \mathrm{D}$

18 percent of sulfur: $30^{\circ} \mathrm{C} ; \dot{\mathrm{O}}, 50^{\circ} \mathrm{C}$.

28 percent of sulfur at $30^{\circ} \mathrm{C}, \Theta$. 


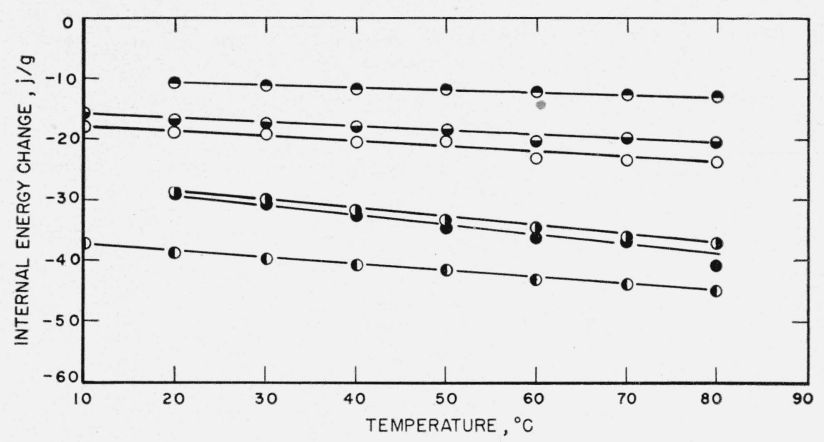

Figure 6. Typical isobaric internal-energy-change behavior for low and high-sulfur contents.

10 percent of sulfur: $, 1,000 \mathrm{~atm} ; \mathbf{D}, 5,000 \mathrm{~atm} ; \bigcirc, 10,000 \mathrm{~atm}$ 28 percent of sulfur: $1,000 \mathrm{~atm} ; \mathrm{J}, 5,000 \mathrm{~atm} ; \mathbf{0}, 10,000 \mathrm{~atm}$

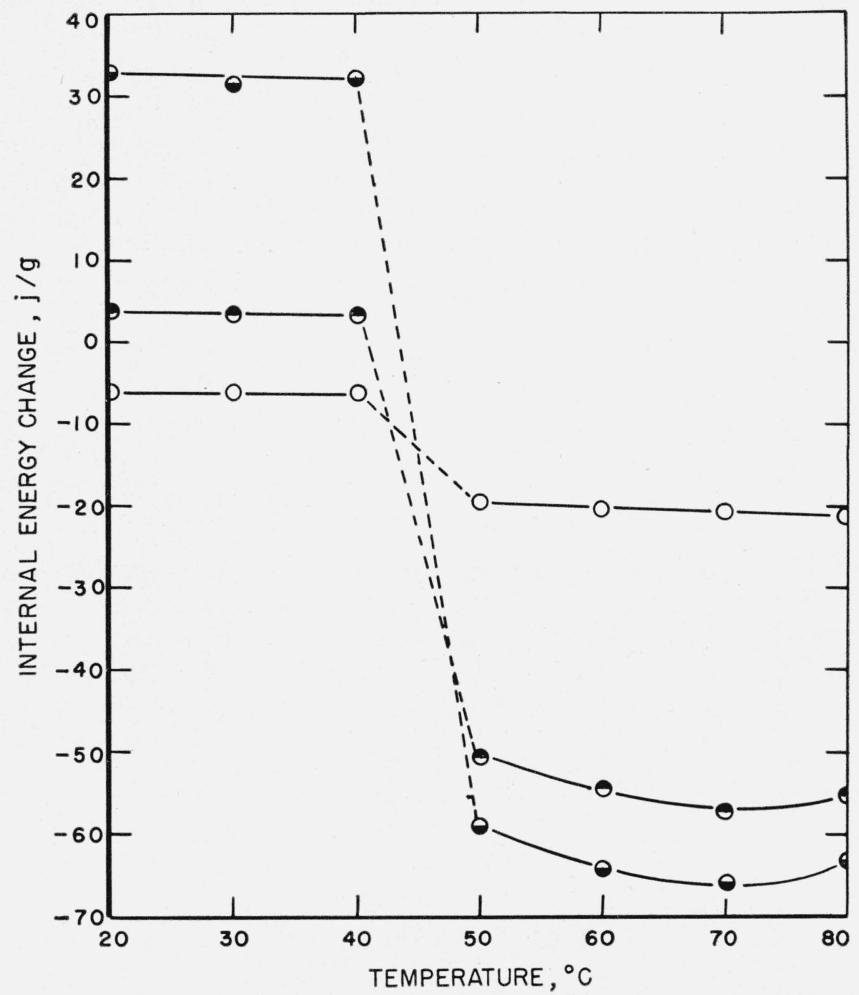

FIGURE 7. Isobaric internal-energy-change behavior at intermediate sulfur content.

18 percent of sulfur: $\bigcirc, 1,000 \mathrm{~atm} ; \boldsymbol{\bullet}, 5,000 \mathrm{~atm} ; \boldsymbol{\bullet}, 10,000 \mathrm{~atm}$.

the lower temperatures is highly suspect as mentioned previously as the expansivities in this region of temperature appeared to possess anomalously high pressure dependence. There is no doubt, however, that at temperatures below $T_{\mathrm{g}}$ the internal energy change reverses sign at a lower pressure than at temperatures above $T_{\mathrm{g}}$. This result also must arise by virtue of the change in $(\partial V / \partial T)_{P}$ at $T_{g}$.

Typical data for internal energy change are shown in figures 5,6 , and 7 .

\section{Change in Heat Capacity}

The change in heat capacity with pressure may be calculated from the relationship $\left(\partial C_{P} / \partial P\right)_{T}=$ $-T\left(\partial^{2} V / \partial T^{2}\right)_{P}$. Because it was previously assumed that $(\partial V / \partial T)_{P}$ was constant within experimental error, such calculations have no basis in fact in these experiments. It may be concluded with assurance that any change in $C_{P}$ with pressure is quite small.

The difference between heat capacities at constant pressure and constant volume may be evaluated from the relationship $C_{P}-C_{V}=-T(\partial V / \partial T)_{P}^{2} /(\partial V / \partial P)_{T}$ which involves only first derivatives. Full data for this difference are not given here. Partial results for two rubbers of interest are given in tables 13 and 14 in terms of specific heats. In table 13, the typical behavior is observed with the difference decreasing rapidly with increasing pressure and approaching a negligible value at high pressures. Isobarically little temperature dependence is noted which follows from the fact that the expansivity was taken as independent of temperature. In table 14, similar behavior is noted above or below $T_{\mathrm{g}}$ but on passing through $T_{\mathrm{g}}$ isobarically a marked change in $C_{P}-C_{V}$ is noted. This result is a direct consequence of the experimental finding that no appreciable change in compressibility occurs at $T_{\mathrm{g}}$ that is analogous to the change in expansivity. The change in the difference between specific heats on passing through $T_{\mathrm{g}}$ amounts to approximately $0.5 \mathrm{j} / \mathrm{g}$. It is probably fortuitous that this figure agrees closely with the change in specific heat at constant pressure for natural rubber observed by Bekkedahl [5] on passing through $T_{\mathrm{g}}$.

TABLE 13. Difference in specific heats at constant pressure and constant volume for rubber-sulfur vulcanizate containing 10 percent of sulfur

\begin{tabular}{|c|c|c|c|c|c|c|c|c|}
\hline \multirow{2}{*}{ Pressure } & \multicolumn{8}{|c|}{$C_{P}-C_{V},(\mathrm{j} / \mathrm{g}) / \operatorname{deg} \mathrm{C}$} \\
\hline & $10^{\circ} \mathrm{C}$ & $20^{\circ} \mathrm{C}$ & $30^{\circ} \mathrm{C}$ & $40^{\circ} \mathrm{C}$ & $50^{\circ} \mathrm{C}$ & $60^{\circ} \mathrm{C}$ & $70^{\circ} \mathrm{C}$ & $80^{\circ} \mathrm{C}$ \\
\hline $\begin{array}{l}\quad \text { atm } \\
1, \ldots \\
1,000 \\
2,000 \\
3,000 \\
4,000 \\
5,000\end{array}$ & $\begin{array}{r}0.40 \\
.32 \\
.22 \\
.22 \\
.15 \\
.09\end{array}$ & $\begin{array}{r}0.40 \\
.31 \\
.22 \\
.22 \\
.14 \\
.09\end{array}$ & $\begin{array}{l}0.39 \\
.31 \\
.22 \\
.21 \\
.14 \\
.08\end{array}$ & $\begin{array}{l}0.38 \\
.30 \\
.22 \\
.21 \\
.13 \\
.08\end{array}$ & $\begin{array}{l}0.38 \\
.30 \\
.22 \\
.21 \\
.13 \\
.08\end{array}$ & $\begin{array}{r}0.37 \\
.30 \\
.22 \\
.21 \\
.13 \\
.08\end{array}$ & $\begin{array}{r}0.36 \\
.30 \\
.22 \\
.20 \\
.13 \\
.08\end{array}$ & $\begin{array}{r}0.36 \\
.30 \\
.22 \\
.20 \\
.12 \\
.08\end{array}$ \\
\hline
\end{tabular}

TABLE 14. Difference in specific heats at constant pressure and constant volume for rubber-sulfur vulcanizate containing 18 percent of sulfur

\begin{tabular}{|c|c|c|c|c|c|c|c|}
\hline \multirow{2}{*}{ Pressure } & \multicolumn{7}{|c|}{$C_{P}-C_{V},(\mathrm{j} / \mathrm{g}) / \operatorname{deg} \mathrm{C}$} \\
\hline & $20^{\circ} \mathrm{C}$ & $30^{\circ} \mathrm{C}$ & $40^{\circ} \mathrm{C}$ & $50^{\circ} \mathrm{C}$ & $60^{\circ} \mathrm{C}$ & $70^{\circ} \mathrm{C}$ & $80^{\circ} \mathrm{C}$ \\
\hline $\begin{array}{l}\text { atm } \\
1,000 \\
2,000 \\
3,000 \\
4,000 \\
5,000\end{array}$ & $\begin{array}{r}0.22 \\
.03 \\
.01 \\
.00 \\
.00 \\
.00\end{array}$ & $\begin{array}{r}0.20 \\
.03 \\
.01 \\
.00 \\
.00 \\
.00\end{array}$ & $\begin{array}{r}0.21 \\
.03 \\
.01 \\
.00 \\
.00 \\
.00\end{array}$ & $\begin{array}{r}0.70 \\
.56 \\
.37 \\
.24 \\
.20 \\
.23\end{array}$ & $\begin{array}{r}0.69 \\
.55 \\
.35 \\
.24 \\
.21 \\
.24\end{array}$ & $\begin{array}{r}0.67 \\
.53 \\
.34 \\
.23 \\
.21 \\
.25\end{array}$ & $\begin{array}{r}0.66 \\
.52 \\
.33 \\
.23 \\
.21 \\
.26\end{array}$ \\
\hline
\end{tabular}


If it is assumed that $C_{P}$ is not materially changed by increasing pressure, further conclusions may be drawn as shown in the following treatment.

It can be shown that the change in the difference of specific heats probably arises from a change in $C_{P}$ and not $C_{V}$ and that $C_{V}$ is, therefore, very probably unchanged on passing through $T_{\mathrm{g}}$. To show this, the derivative of the difference of specific heats with respect to temperature is taken and is found to be equal to

$$
\begin{gathered}
\frac{\Delta\left(C_{P}-C_{V}\right)}{\Delta T}=\left(C_{P}-C_{V}\right)\left[\frac{1}{T}+2\left(\frac{\partial^{2} V}{\partial T^{2}}\right)_{P} /\left(\frac{\partial V}{\partial T}\right)_{P}-\right. \\
\left.\left(\frac{\partial^{2} V}{\partial P \partial T}\right)_{T \cdot P} /\left(\frac{\partial V}{\partial P}\right)_{T}\right] .
\end{gathered}
$$

On considering the terms in the bracket, it can be concluded that in a small temperature interval including $T_{\mathrm{g}}, 1 / T$ is negligible. It has been shown [2] that the temperature coefficient of compressibility is small compared to the compressibility, so that the third term is also negligible. It is known, however, that the temperature coefficient of expansivity is large in this temperature interval so that the ratio of $\left(\partial^{2} V / \partial T^{2}\right) /(\partial V / \partial T)$ is of the order of $\frac{1}{2}$ or $\frac{1}{3}$ in this interval. Therefore any change of $C_{P}-C_{V}$ on passing through $T_{\mathrm{g}}$ arises predominantly from a change in expansivity. Because the basic distinc. tion betweeo $C_{P}$ and $C_{V}$ involves this expansivity, it follows that $C_{\mathrm{V}}$ is most probably unaffected by passing through $T_{\mathrm{g}}$, except for the normal small variation to be expected to arise from temperature changes alone. Although the suggested constancy of $C_{V}$ on passing through $T_{\mathrm{g}}$ must have significance as regards the processes responsible for the changes at $T_{\mathrm{g}}$, no possible explanations of this behavior can be proposed at this time.

Using the data presented, it is possible to calculate $C_{V}$ by means of the relationship

$$
C_{V} \equiv\left(\frac{\partial E}{\partial T}\right)_{V}=\left(\frac{\partial E}{\partial T}\right)_{P}+\left(\frac{\partial E}{\partial P}\right)_{T}\left(\frac{\partial P}{\partial T}\right)_{V},
$$

where $E$ is the internal energy. All terms on the right side of this equation are obtainable from the present data. However, attempts to verify the suggested constancy of $C_{V}$ in passing through $T_{\mathrm{g}}$, or to calculate $C_{V}$ for any specimen were unsuccessful. The reasons for this failure are to be attributed to the predominant effect of the expansivity integral in determining the values of $\Delta E$, and the expansivity itself in determining the values of $(\partial P / \partial T)_{V}$. As pointed out previously, the expansivity is not believed to be sufficiently well determined to justify reliance on the results of such calculations.

\section{Pressure Coefficient}

The pressure coefficient $-(\partial P / \partial T)_{V}$ may be calculated from the ratio of expansivity to compressibility. There seems little point in reproducing these values which are generally of the order of 10 to 20 atm per $\operatorname{deg} \mathrm{C}$. It is to be noted, however, that by virtue of the change in expansivity, the pressure coefficient is discontinuous at $T_{\mathrm{g}}$. Sufficient data are presented here to permit the calculation of these values if they are of interest.

\section{Discussion}

The data presented for three typical rubbers of high sulfur content are believed to be representative of the PVT data obtained here. Calculations of a similar nature have not been carried out on all samples tested due to the laborious nature of the calculations and the fact that the other samples yielded quite similar experimental results. The calculations described above have been carried through with one other rubber containing 22 percent of sulfur with results similar in all important respects to those obtained on the 18-percent-sulfur vulcanizate described here. Specific qualifications concerning the numerical accuracy of the data have been emphasized, but significant behavior of the calculated quantities has been shown to be a direct consequence of the experimental observations where $T_{\mathrm{g}}$ is involved or to agree otherwise with the results obtained by Bridgman [3] for solids and liquids. While the general trends of most quantities reported are believed to be of interest, it would appear that the most significant result lies in the suggestion that $C_{V}$ very probably does not change discontinuously at $T_{\mathrm{g}}$. Inasmuch as significant differences may exist in glass-transition temperatures found by Scott [4] and in the present data, it is planned to measure the specific volume - temperature relationships of the specimens used in these studies, using dilatometers at $1 \mathrm{~atm}$ and modifications of the present high pressure equipment at elevated pressures. The values so obtained will be used to recalculate portions of the data presented here. Any significant differences will be reported at a future date.

\section{References}

[1] C. E. Weir, J. Research NBS 50, 3 (1953) RP2403.

[2] C. E. Weir, J. Research NBS 50, 6 (1953) RP2421.

[3] P. W. Bridgman, The physics of high pressure (G. Bell \& Sons, Ltd, London 1949).

[4] A. H. Scott, J. Research NBS 14, 100 (1935) RP760.

[5] N. Bekkedahl and H. Matheson, J. Research NBS 15, 503 (1935) RP844.

Washington, March 6, 1953. 